\title{
THE SMOOTH INVARIANCE OF THE KODAIRA DIMENSION OF A COMPLEX SURFACE
}

\author{
Robert Friedman And Zhenbo Qin
}

\section{Introduction}

The purpose of this note is to announce the following result:

Theorem 1. Let $X$ be a complex surface of general type. Then $X$ is not diffeomorphic to a rational surface.

Combining Theorem 1 with the results in [5] gives:

Corollary 2. If $X$ is a complex surface diffeomorphic to a rational surface, then $X$ is a rational surface. Thus, up to deformation equivalence, there is a unique complex structure on the smooth 4-manifolds $S^{2} \times S^{2}$ and $\mathbb{C} P^{2} \# n \overline{\mathbb{C} P}^{2}$.

On the other hand, using the results of [7], we obtain:

Corollary 3. If $X_{1}$ and $X_{2}$ are two diffeomorphic complex surfaces, then

$$
\kappa\left(X_{1}\right)=\kappa\left(X_{2}\right)
$$

where $\kappa\left(X_{i}\right)$ denotes the Kodaira dimension of $X_{i}$.

Corollary 3 settles a conjecture due to Van de Ven [21] (see also [6]).

Let us give a brief history of results along these lines. Yau [23] showed that every complex surface homotopy equivalent to $\mathbb{C} P^{2}$ is in fact $\mathbb{C} P^{2}$. In [3], Donaldson used gauge theory to show that a certain simply connected elliptic surface known to be homeomorphic to the blowup of $\mathbb{C} P^{2}$ at nine points was not in fact diffeomorphic to a rational surface. In [5] this result was generalized to show that no nonrational elliptic surface could be diffeomorphic to a rational surface. (The case of minimal elliptic surfaces is also considered in [15]). The methods developed in [5] also characterize the possible self-diffeomorphisms of rational surfaces. It follows from the

Received February 17, 1994.

The first author was partially supported by NSF grant DMS-9203940. 
work of Kotschick, Okonek-Van de Ven, and Pidstrigach [9, 11, 16, 17] that no minimal surface of general type oriented homotopy equivalent to $\mathbb{C} P^{2} \# 8 \overline{\mathbb{C P}}^{2}$ can be diffeomorphic to a rational surface, and Kotschick [11] has announced a similar result for the blowups of such a surface. According to $[19,20]$, surfaces of general type whose second Betti number are at most 10 and whose algebraic minimal models do not contain certain smooth rational curves can not be diffeomorphic to rational surfaces; in particular, this result handles the case where $X$ is oriented homotopy equivalent to $S^{2} \times S^{2}$ or $\mathbb{C} P^{2} \# \overline{\mathbb{C P}}^{2}$. At the same time, building on ideas of Donaldson, Pidstrigach and Tyurin [18], using Spin polynomial invariants, showed that no minimal surface of general type is diffeomorphic to a rational surface. We shall outline a new proof of the theorem of Pidstrigach-Tyurin below, which will give extra information on the possible embedded 2-spheres in $X$.

\section{Preliminaries on $S O(3)$-invariants}

Let $X$ be a smooth simply connected 4-manifold with $b_{2}^{+}(X)=1$, and fix an $S O(3)$-bundle $P$ over $X$ with $w_{2}(P)=w$ and $p_{1}(P)=p$. Then, a wall of type $(w, p)$ for $X$ is a class $\zeta \in H^{2}(X ; \mathbb{Z})$ such that $\zeta \equiv w \bmod 2$ and $p \leq \zeta^{2}<0$. Let

$$
\Omega_{X}=\left\{x \in H^{2}(X ; \mathbb{R}): x^{2}>0\right\}
$$

be the positive cone of $X$, and let $W^{\zeta}=\Omega_{X} \cap(\zeta)^{\perp}$. A chamber of type $(w, p)$ for $X$ is a connected component of the set

$$
\Omega_{X}-\bigcup\left\{W^{\zeta}: \zeta \text { is a wall of type }(w, p)\right\} .
$$

For a chamber $\mathcal{C}$ of type $(w, p)$ on $X$, let $\gamma_{w, p}(X ; \mathcal{C}) \in \operatorname{Sym}^{d(p)}\left(H^{2}(X ; \mathbb{Z})\right)$ be the Donaldson polynomial invariant associated to the $S O(3)$-bundle $P$ and the chamber $\mathcal{C}$ defined in $[10,12]$, where $d(p)=-p-3$. When $X$ is an algebraic surface and $L$ is an ample divisor on $X$, let $c_{1}$ be a divisor whose mod two reduction is $w$ and let $c_{2}$ be the integer defined by $p=c_{1}^{2}-4 c_{2}$. If $\mathfrak{M}_{L}\left(c_{1}, c_{2}\right)$ denotes the moduli space of $L$-stable rank-2 holomorphic vector bundles $V$ over $X$ with $c_{1}(V)=c_{1}$ and $c_{2}(V)=c_{2}$, then $\gamma_{w, p}(X ; \mathcal{C})$ can be computed from $\mathfrak{M}_{L}\left(c_{1}, c_{2}\right)$ in certain circumstances $[4,7]$. Our first result states that, under certain nonvanishing hypotheses for $\gamma_{w, p}(X ; \mathcal{C})$, certain classes in $H^{2}(X ; \mathbb{Z})$ cannot be represented by smoothly embedded 2-spheres.

Theorem 4. Let $e \in H^{2}(X ; \mathbb{Z})$ be the cohomology class of a smoothly embedded 2-sphere in $X$ with $e^{2}=-1$, and let $(w \cdot e)$ be odd. Suppose that 
$M \in H_{2}(X ; \mathbb{Z})$ satisfies $M^{2}>0$ and $M \cdot e=0$. Then, for every chamber $\mathcal{C}$ of type $(w, p)$ whose closure contains $M$,

$$
\gamma_{w, p}(X ; \mathcal{C})\left(M^{d}\right)=0
$$

In case $e$ is not a wall of the chamber $\mathcal{C}$, which is the only case necessary for this paper, Theorem 4 is due to Kotschick [10].

We shall play Theorem 4 off against the following nonvanishing theorem: let $M$ be a divisor on the algebraic surface $X$ such that, for all $k \gg 0$, the linear system $|k M|$ has no base points and defines a birational morphism $\varphi: X \rightarrow \bar{X}$ to a normal surface $\bar{X}$. We assume further that $X$ is minimal, or more generally that there are no exceptional curves $E$ of the first kind contracted by $\varphi$, i.e. such that $M \cdot E=0$.

Theorem 5. Let $w \in H^{2}(X ; \mathbb{Z} / 2 \mathbb{Z})$ be the mod two reduction of $\left[K_{X}\right]$. Suppose that the connected components of the set of one-dimensional fibers of $\varphi$ are either irreducible curves or the minimal resolutions of rational or minimally elliptic singularities. Let $\mathcal{C}$ be a chamber of type $(w, p)$ containing $M$ in its closure which contains the first Chern class of an ample line bundle. Then for all $p \ll 0$, if $d=-p-3$, then

$$
\gamma_{w, p}(X ; \mathcal{C})\left(M^{d}\right)>0
$$

Theorem 5 is a generalization of Donaldson's nonvanishing theorem for ample divisors on algebraic surfaces with positive geometric genus $[2,4$, 7 Chapter 5], and the proof uses very similar ideas together with work of Jun Li $[13,14]$. We note that the statement and proof of Theorems 4 and 5 carries over to the case where $p_{g}(X)>0$ as well, where we can drop the chamber $\mathcal{C}$ from the notation.

Combining Theorem 4 and Theorem 5 , we see that, if there is a class $E$ of square -1 in $H^{2}(X ; \mathbb{Z})$ such that $M \cdot E=0$, where $M$ satisfies the hypotheses of Theorem 5, then $E$ cannot be represented by an embedded 2 -sphere. In the next section, given a class $E$ with $E^{2}=-1$ and $E \cdot K_{X}=$ 1 , we shall explain how to find divisors $M$ on minimal simply connected surfaces $X$ of general type which satisfy the hypotheses of Theorem 5 and such that $M \cdot E=0$.

\section{Embedded spheres in minimal surfaces of general type}

Our main result concerning minimal surfaces is the following base point free theorem: 
Theorem 6. Let $X$ be a minimal simply connected surface of general type. Let $E$ be a $(1,1)$-class in $H^{2}(X ; \mathbb{Z})$ such that $E^{2}=-1$ and $E \cdot K_{X}=1$. Then there exists an orientation-preserving diffeomorphism $\psi: X \rightarrow X$ and a divisor $M$ on $X$ such that

(i) $M \cdot \psi^{*} E=0$;

(ii) $|k M|$ is base-point-free for all $k \gg 0$ and defines a birational morphism $\varphi: X \rightarrow \bar{X}$ which satisfies the hypotheses of Theorem 5 .

Note that, if $p_{g}(X)=0$, then every class is a $(1,1)$-class. If $p_{g}(X)>$ 0 and $E$ is a class of square -1 in $H^{2}(X ; \mathbb{Z})$ which is represented by a smoothly embedded 2-sphere, then it follows from a result of Brussee [1] that $E$ is a $(1,1)$-class.

Given Theorem 6, let us show how to deduce Theorem 1 for minimal surfaces $X$ of the same homotopy type as a rational surface. By applying Theorems 4 and 5 we conclude that the class $\psi^{*} E$ cannot be represented by a smoothly embedded 2 -sphere. Likewise $E$ itself cannot be represented by a smoothly embedded 2-sphere. Now there is an oriented homotopy equivalence $\alpha$ from $X$ to a del Pezzo surface $Y$. Using a result of Kneser [8], we may further assume that $\alpha^{*}\left[K_{Y}\right]=-\left[K_{X}\right]$. If $X$ is homotopy equivalent to $S^{2} \times S^{2}$, we are done by the results of [19]. If $Y \neq S^{2} \times S^{2}$, then there exist classes $E^{\prime}$ on $Y$ satisfying $\left(E^{\prime}\right)^{2}=E^{\prime} \cdot K_{Y}=-1$ (the classes of exceptional curves). Setting $E=\alpha^{*} E^{\prime}$, we see that there exist classes $E$ satisfying the hypotheses of Theorem 6 on $X$. Moreover, it is a consequence of a theorem of Wall [22] that, if $X$ is diffeomorphic to a rational surface, then these classes are represented by embedded 2-spheres, a contradiction. Thus $X$ cannot be diffeomorphic to a rational surface.

The above argument should be contrasted with the method of $[19,20]$ to show that $X$ is not diffeomorphic to a rational surface. There it is shown that, for an appropriate choice of a basis $\left\{E_{0}, \ldots, E_{n}\right\}$ for $H^{2}(X ; \mathbb{Z})$, with $E_{0}^{2}=1$ and $E_{i}^{2}=-1$ for $i>0$ and for $w$ the $\bmod 2$ reduction of $E_{i}$, the polynomial invariant is not zero for a suitable chamber. On the other hand, for a del Pezzo surface $Y$ and for the corresponding class and chamber, the invariant is zero, as can be seen by an easy calculation with stable bundles. Roughly speaking, the underlying idea is that, if the polynomial invariant is nonzero, then the class $E_{0}-E_{i}$ of square zero cannot be represented by a smoothly embedded 2-sphere.

Let us give a very brief idea of the proof of Theorem 6. First suppose that the divisor $M=K_{X}+E$ is nef. Note that $M \cdot E=0$. Consider the curves $C$ such that $M \cdot C=0$ and let $X^{\prime}$ be the surface obtained by contracting these curves (which have a negative definite intersection matrix, since $M$ has positive square). If the collection of these curves is the resolution 
of a rational singularity, then $M$ induces an ample divisor on $X^{\prime}$ by the Nakai-Moishezon criterion and so $M$ is eventually base point free. Thus $M$ is a divisor to which we can apply Theorem 5 . If there is a nonrational singular point on $X^{\prime}$, then we show that there is an effective rational linear combination $\sum_{i} a_{i} C_{i}$ of the curves contracted by $M$ such that the $\mathbb{Q}$-divisor $K_{X}+\sum_{i} a_{i} C_{i}$ is orthogonal to $E$, nef and big and eventually base point free, and such that the image of the resulting contraction has only rational and minimally elliptic singularities. Thus we can again apply Theorem 5 . Finally, there is the case where $M$ is not nef. In this case, we claim that after modifying $E$ by a suitable orientation-preserving self-diffeomorphism $\psi$ of $X$, we can assume that $M \cdot C \geq 0$ for all smooth rational curves $C$ on $X$ of self-intersection -2 . Indeed, the reflections about the classes of such curves are realized by orientation-preserving self-diffeomorphisms of $X$, and it is well-known that after applying an element in the group generated by these reflections we can arrange that $E \cdot C \geq 0$ for all such $C$. Thus a curve $C$ satisfying $M \cdot C<0$ either has $p_{a}(C) \geq 1$ or $p_{a}(C)=0$ and $C^{2} \leq-3$ (since $X$ is minimal and by the above construction). In either case it is easy to find a $\mathbb{Q}$-divisor of the form $K_{X}+\lambda C$ with $\lambda>0$ which is nef, big, eventually base point free, and orthogonal to $E$, and we are done in this case as well.

The arguments used to prove Theorem 6 together with the known results for $S^{2} \times S^{2}$ also show the following:

Theorem 7. Let $X$ be a minimal surface of general type oriented homotopy equivalent to a rational surface, and let $K_{X}$ be the canonical class of $X$. Then there exist

(i) a class $w \in H^{2}(X ; \mathbb{Z} / 2 \mathbb{Z})$;

(ii) an integer $p \in \mathbb{Z}$;

(iii) a chamber $\mathcal{C}$ for $X$ of type $(w, p)$, and

(iv) a homotopy equivalence $\alpha: Y \rightarrow X$, where $Y$ is a suitable blowup of $\mathbb{C} P^{2}$ at distinct points, satisfying $\left[K_{Y}\right]=-\alpha^{*}\left[K_{X}\right]$,

such that

$$
\alpha^{*} \gamma_{w, p}(X ; \mathcal{C}) \neq \pm \gamma_{\alpha^{*} w, p}\left(Y, \alpha^{*} \mathcal{C}\right)
$$

\section{Reduction to the minimal case}

To complete the proof of Theorem 1, we must handle the case of a nonminimal surface $X$. We begin by recalling some terminology and results from [5]. A good generic rational surface $Y$ is a rational surface such that $K_{Y}=-C$ where $C$ is a smooth curve, and such that there does not exist a smooth rational curve on $Y$ with self-intersection -2 . Every rational 
surface is diffeomorphic to a good generic rational surface. If $Y$ is a good generic rational surface with $b_{2}^{-}(Y) \leq 8$, then $Y$ is a del Pezzo surface.

Now suppose that some blow up $\tilde{X}$ of a minimal surface of general type is diffeomorphic to a rational surface $\tilde{Y}$, which we may assume to be a good generic rational surface. Then the exceptional classes in $\tilde{X}$ yield smoothly embedded 2 -spheres of self-intersection -1 in $\tilde{Y}$. Thus the reflection about the class of each such 2 -sphere is realized by a selfdiffeomorphism of $\tilde{Y}$. Now the possible automorphisms of $H^{2}(\tilde{Y} ; \mathbb{Z})$ realized by self-diffeomorphisms of $\tilde{Y}$ are described in [5] using the chamber structure on $H^{2}(\tilde{Y} ; \mathbb{Z})$ arising from walls of square -1 . With this description, and the general theory developed in [5], we can show the following:

Theorem 8. Let $X$ be a minimal surface of general type and let $\tilde{X} \rightarrow X$ be a blowup of $X$ at $r$ distinct points. Let $E_{1}^{\prime}, \ldots, E_{r}^{\prime}$ be the homology classes of the exceptional curves on $\tilde{X}$. Let $\psi_{0}: \tilde{X} \rightarrow \tilde{Y}$ be a diffeomorphism, where $\tilde{Y}$ is a good generic rational surface. Then there exist a diffeomorphism $\psi: \tilde{X} \rightarrow \tilde{Y}$ and a good generic rational surface $Y$ with the following properties:

(i) The surface $\tilde{Y}$ is the blowup of $Y$ at $r$ distinct points.

(ii) If $f_{1}, \ldots, f_{r}$ are the classes of the exceptional curves in $H^{2}(\tilde{Y} ; \mathbb{Z})$, then possibly after renumbering $\psi^{*}\left(f_{i}\right)=E_{i}^{\prime}$ for all $i$.

(iii) Identifying $H^{2}(X)$ with a subgroup of $H^{2}(\tilde{X})$ and $H^{2}(Y)$ with a subgroup of $H^{2}(\tilde{Y})$ in the obvious way, we have $\psi^{*}\left(H^{2}(Y)\right)=H^{2}(X)$.

Moreover, for every choice of an isometry $\tau$ from $H^{2}(Y)$ to $H^{2}(X)$, there exists a choice of a diffeomorphism $\psi$ satisfying $(i)-($ iii $)$ above and such that $\psi^{*} \mid H^{2}(Y)=\tau$.

In particular, if $\tilde{X}$ is a surface of general type diffeomorphic to a rational surface $\tilde{Y}$, we can choose the diffeomorphism so as to line up the homology classes of the exceptional curves. Now we have the following "easy" blow up formula:

Lemma 9. Let $X \# \overline{\mathbb{C P}}^{2}$ be a blowup of $X$, and identify $H_{2}(X ; \mathbb{Z})$ with a subspace of $H_{2}\left(X \# \overline{\mathbb{C P}}^{2} ; \mathbb{Z}\right)$ in the natural way. Given $w \in H^{2}(X ; \mathbb{Z} / 2 \mathbb{Z})$, let $\tilde{\mathcal{C}}$ be a chamber of type $(w, p)$ for $X \# \overline{\mathbb{C} P}^{2}$ containing the chamber $\mathcal{C}$ in its closure. Then

$$
\gamma_{w, p}\left(X \# \overline{\mathbb{C} P}^{2} ; \tilde{\mathcal{C}}\right) \mid H_{2}(X ; \mathbb{Z})= \pm \gamma_{w, p}(X ; \mathcal{C})
$$

Combining Theorem 8 and Lemma 9, it is easy to see that we can arrange a contradiction to Theorem 7 . 
One should interpret Theorem 8 as follows: if we can distinguish the diffeomorphism type of minimal surfaces $X$ from rational surfaces by an invariant which satisfies an "easy" blow up formula, then we can also prove that nonminimal surfaces of general type cannot be diffeomorphic to rational surfaces. In particular, using an "easy" blow up formula for the Spin polynomials, we could give a different proof of Theorem 1 based on the results of Pidstrigach-Tyurin. However, one goal of this research has been to find an independent proof of the theorem of Pidstrigach-Tyurin using only the $S O(3)$-invariants. Aside from the desirability of a different proof, our method also gives some new information on the possible smoothly embedded 2-spheres in a simply connected surface of general type. Indeed the arguments outlined above prove the following:

Theorem 10. Let $X$ be a minimal simply connected surface of general type. Let $E \in H^{2}(X ; \mathbb{Z})$ satisfy $E^{2}=-1$ and $E \cdot K_{X}=1$. Let $\tilde{X}$ be a blowup of $X$, and view $H^{2}(X ; \mathbb{Z})$ as included in $H^{2}(\tilde{X} ; \mathbb{Z})$ in the natural way. Then the class $E \in H^{2}(\tilde{X} ; \mathbb{Z})$ is not represented by a smoothly embedded 2 -sphere.

\section{Acknowledgements}

The second author would like to thank Professors Sheldon Katz and Jun Li for valuable help and stimulating discussions.

\section{References}

1. R. Brussee, On the (-1)-curve conjecture of Friedman and Morgan, Invent. Math. 114 (1993), 219-229.

2. S. K. Donaldson, Anti-self-dual Yang-Mills connections over complex algebraic surfaces and stable vector bundles., Proc. Lond. Math. Soc. 50 (1985), 1-26.

3. I Irrationality and the h-cobordism conjecture, J. Differ. Geom. 26 (1987), 141-168.

4. __ Polynomial invariants for smooth four-manifolds, Topology 29 (1990), 257315.

5. R. Friedman and J. W. Morgan, On the diffeomorphism types of certain algebraic surfaces, I. J. Differ. Geom. 27 (1988), 297-369.

6. _ Algebraic surfaces and 4-manifolds: some conjectures and speculations, Bull. Amer. Math. Soc. (N.S.) 18 (1988), 1-19.

7. __ Smooth four-manifolds and complex surfaces, Ergebnisse der Mathematik und ihrer Grenzgebiete 3 Folge, Springer, Heidelberg, Berlin, New York, 1994.

8. M. Kneser, Klassenzahlen indefiniter quadratischer Formen in drei oder mehr Veränderlichen, Arch. der Math. 7 (1956), 323-332.

9. D. Kotschick, On manifolds homeomorphic to $\mathbb{C} P^{2} \# 8 \overline{\mathbb{C} P}^{2}$, Invent. Math. 95 (1989), 591-600.

10. (1991), 426-448.

11. _ Positivity versus rationality of algebraic surfaces, in preparation. 
12. D. Kotschick and J. W. Morgan, $S O(3)$-invariants for 4-manifolds with $b_{2}^{+}=1$, II, to appear in J. Differ. Geom.

13. J. Li, Algebraic geometric interpretation of Donaldson's polynomial invariants, J. Differ. Geom. 37 (1993), 417-466.

14. Kodaira dimension of moduli space of vector bundles on surfaces, Invent. Math. 115 (1994), 1-40.

15. C. Okonek and A. Van de Ven, Stable bundles and differentiable structures on certain elliptic surfaces, Invent. Math. 86 (1986), 357-370.

16. _ ture of Barlow's surface, Invent. Math. 95 (1989), 602-614.

17. V. Y. Pidstrigach, Deformation of instanton surfaces, Math. USSR Izvestiya 38 (1992), 313-331.

18. V. Y. Pidstrigach and A. N. Tyurin, Invariants of the smooth structure of an algebraic surface arising from the Dirac operator, Russian Academy of Science Izvestiya Mathematics (Translations of the AMS) 40 (1993), 267-351.

19. Z. B. Qin, Complex structures on certain differentiable 4-manifolds, Topology 32 (1993), 551-566.

20. __ On smooth structures of potential surfaces of general type homeomorphic to rational surfaces, Invent. Math. 113 (1993), 163-175.

21. A. Van de Ven, On the differentiable structure of certain algebraic surfaces, Sém. Bourbaki ${ }^{\circ}$ 667, Juin 1986.

22. C. T. C. Wall, Diffeomorphisms of 4-manifolds, J. Lond. Math. Soc. 39 (1964), 131-140.

23. S. T. Yau, On the Ricci curvature of compact Kähler manifolds and the complex Monge-Ampère equation, Comm. Pure Appl. Math. 31 (1978), 339-411.

Department of Mathematics, Columbia University, New York, NY 10027, USA

E-mail address: rf@math.columbia.edu

Department of Mathematics, Oklahoma State University, Stillwater, OK 74078, USA

E-mail address: qz@math.okstate.edu 